\title{
Estado nutricional de portadores de doença renal crônica em hemodiálise no Sistema Único de Saúde
}

\author{
Nutritional status of individuals with chronic renal failure \\ in hemodialysis in the Unified Health System
}

Kelb Bousquet-Santos ${ }^{1}$

Luciane da Graça da Costa ${ }^{1}$

Joanlise Marco De Leon Andrade 2

${ }^{1}$ Programa de PósGraduação em Ciências e Tecnologias em Saúde, Faculdade Ceilândia, Universidade de Brasília Campus Universitário Centro Metropolitano Ceilândia Sul, Ceilândia. 72220-275 Brasília DF Brasil.kelb@unb.br ${ }^{2}$ Departamento de Estatística, Instituto de Ciências Exatas, Universidade de Brasília. Brasília DF Brasil.

\begin{abstract}
Malnutrition is a major factor in the evolution of patients with chronic kidney disease (CKD) on hemodialysis (HD). It is associated with increased frequency and the duration of hospitalizations. The scope of this study was to evaluate the nutritional status of patients with CKD on HD in public or private clinics of the Unified Health System in the Federal District. Sociodemographic, biochemical and anthropometric data were obtained from 96 participants, 35.5\% of which were from public clinics. Parameters for evaluation of malnutrition in CKD included Body Mass Index $(B M I)<23 \mathrm{~kg} / \mathrm{m}^{2}$, reduced arm muscle circumference (AMC) and serum albumin $<3.8 \mathrm{~g} / \mathrm{dL}$. From all participants, $14.6 \%$ were classified as malnourished (60 \pm 12 years; 57\% male; $69 \%$ private clinics); $33.3 \%$ presented adequate nutritional status (55 \pm 14 years; 53\% male; $57 \%$ private clinics); $52.1 \%$ had at least one parameter related to malnutrition. BMI below the recommended parameter was the most prevalent variable (42.7\%), followed by reduced AMC (41.7\%) and serum albumin (33.3\%). Based on these results, it may be concluded that at least one positive variable was related to malnutrition in half of the sample, which reinforces the importance of nutritional assessment in the context of the treatment of CKD.
\end{abstract}

Key words Malnutrition, Unified Health System, Chronic kidney disease, Kidney dialysis
Resumo A desnutrição é um fator relevante na evolução dos pacientes com doença renal crônica (DRC) em hemodiálise (HD), estando associada a maior frequência e duração de internações hospitalares. Objetivou-se avaliar o estado nutricional de pacientes com DRC em HD em unidades públicas ou privadas vinculadas ao SUS no Distrito Federal. Dados sociodemográficos, bioquímicos e antropométricos foram obtidos de 96 participantes, sendo $35,5 \%$ provenientes de hospitais públicos. Os critérios utilizados para avaliação de desnutrição na DRC foram Índice de Massa Corpórea $(I M C)<23 \mathrm{~kg} / \mathrm{m}^{2}$, circunferência muscular do braço $(C M B)$ reduzida e albumina sérica < $3,8 \mathrm{~g} / \mathrm{dl}$. Do total de avaliados, $14,6 \%$ apresentou desnutrição (60 \pm 12 anos; 57\% masculino; 69\% clínicas privadas); 33,3\% apresentou estado nutricional adequado (55 \pm 14 anos; 53\% masculino; $57 \%$ clinicas privadas); $52,1 \%$ tinha ao menos uma variável relacionada à desnutrição, onde o IMC abaixo do recomendado foi o mais prevalente $(42,7 \%)$, seguido da CMB reduzida (41,7\%) e da albumina sérica (33,3\%). Com base nos resultados conclui-se que ao menos uma variável relacionada ao estado nutricional esteve alterada em metade da amostra estudada, o que reforça a importância da avaliação nutricional no contexto do tratamento da DRC.

Palavras-chave Desnutrição, SUS, Insuficiência renal crônica, Diálise renal 


\section{Introdução}

A Doença Renal Crônica (DRC) é um problema de saúde pública com impacto econômico e social significativo. Em 1990, a DRC representava a $17^{\mathrm{a}}$ causa de morte no Brasil, enquanto em 2010 já ocupava a $10^{\mathrm{a}}$ colocação. Além disso, observou-se um aumento expressivo na contribuição da DRC como causa de morte prematura em mulheres brasileiras $^{1}$. A hipertensão e o diabetes seguem como as principais causas de DRC que causam falência dos rins e necessidade de terapia renal substitutiva, o que acarreta em enorme ônus ao sistema de saúde. Dentre os tipos de terapia substitutiva, a hemodiálise (HD) representa a principal escolha, sendo utilizada em $91 \%$ dos casos $^{2}$. O Registro Latino-Americano de Diálise mostra um aumento na prevalência de portadores de DRC em uso de terapia renal substitutiva, em particular nos países que têm uma cobertura universal de saúde pública, sendo o Brasil o país com maior número de pacientes nesta condição ${ }^{3}$. A taxa real de tratamento renal substitutivo no Brasil continua inferior a encontrada em países como o Chile, Argentina, de alguns países desenvolvidos da Europa e também da norte americana. Entretanto, há grandes variações regionais no Brasil, com valores obtidos nas regiões Sudeste e Sul próximos aos encontrados em países desenvolvidos ${ }^{3}$.

A depleção do estado nutricional é frequentemente observada à medida que a perda da função renal evolui. A etiologia da desnutrição associada à DRC é multifatorial, sendo quase sempre associada à ingestão alimentar insuficiente e/ou, principalmente, ao hipercatabolismo, além das perdas de nutrientes durante o procedimento hemodialítico ${ }^{4}$. A desnutrição possui um impacto negativo na evolução dos pacientes em HD pois está associada a um maior número de complicações, como maior risco de infecções, além de maior frequência e duração nas internações hospitalares ${ }^{5}$. Estudos nacionais apresentam uma ampla variação na prevalência de desnutrição moderada a grave associada a HD, possivelmente em função das diferentes metodologias utilizadas na sua avaliação ${ }^{6,7}$.

A monitoração periódica do estado nutricional deve fazer parte do acompanhamento de pacientes em diálise, sendo fundamental para prevenir, diagnosticar e tratar a desnutrição. Apesar da importância do estado nutricional na evolução do paciente com DRC em tratamento hemodialítico, apenas em 2010 foi realizado o $1^{\circ}$ Censo do Estado Nutricional de Pacientes em HD pela Sociedade Brasileira de Nefrologia $(\mathrm{SBN})^{8}$. Dentre os resultados obtidos observou-se que $44,6 \%$ dos pacientes avaliados realizavam HD pelo Sistema Único de Saúde (SUS), os quais apresentaram maior prevalência de eutrofia e níveis adequados de albumina sérica em comparação aos pacientes atendidos por convênio. No entanto, apenas 36 (12\%) das 626 Unidades Renais Cadastradas na SBN e ativas concordaram em participar do censo, sendo 17 na região Sudeste, nove na região Sul, cinco na região Centro-Oeste, quatro na região Nordeste e apenas uma na região Norte. Além disso, dados de apenas 50\% dos pacientes destas unidades foram enviados e contabilizados no Censo. Em toda a região Centro-Oeste, cinco unidades de diálise responderam ao Censo, totalizando 271 pacientes, ficando apenas à frente da Região Norte $(n=75)$ em número de pacientes incluídos no estudo, o que reforça a necessidade de estudos que avaliem o perfil nutricional desse grupo nessa região brasileira. Especula-se que os pacientes tratados nos estabelecimentos que não responderam estejam em situação de saúde mais precária, especialmente em algumas regiões menos favorecidas do país. Sendo assim, os resultados apresentados no censo nutricional da SBN demonstram a necessidade premente de informações acerca do estado nutricional de pacientes em HD naquelas regiões brasileiras onde observou-se maior escassez de informação, ou seja, nas regiões Centro-Oeste, Nordeste e Norte.

Diante deste contexto e considerando que a identificação e o tratamento precoce do déficit nutricional durante o tratamento hemodialítico podem reduzir o risco de infecções e outras complicações, bem como a mortalidade nesses pacientes, o objetivo do presente estudo foi avaliar o estado nutricional de pacientes com DRC em HD em unidades públicas ou privadas vinculadas ao SUS no Distrito Federal.

\section{Metodologia}

Trata-se de um estudo transversal com amostra por conveniência. Com base no Cadastro Nacional de Estabelecimentos de Saúde foram selecionados cinco centros de HD do Distrito Federal, de gestão pública ou privada, que prestassem serviço para a população adulta proveniente do SUS, e que concordassem em participar do estudo. Do total de 16 centros de HD cadastrados (11 privados e quatro públicos), um não concordou em participar do estudo; quatro centros de gestão privada não prestavam serviço ao SUS; um centro de gestão pública prestava serviço exclusivamente para pacientes portadores de HIV. Sen- 
do assim, dos 10 centros de HD restantes, cinco foram selecionados para o estudo (três de gestão privada e dois de gestão pública). A escolha pelas clínicas deu-se por conveniência, de forma que, das clínicas elegíveis, fossem aquelas em diferentes regiões administrativas do DF. A opção de estudo dos pacientes exclusivamente do SUS deu-se pelo fato de o número de sessões de HD semanais diferir entre esses pacientes e aqueles atendidos por convênio. Os critérios de inclusão do estudo foram: ter idade igual ou superior a 18 anos e estar em tratamento hemodialítico há pelo menos três meses. Os critérios de exclusão foram: ser portador de patologia que afetasse diretamente o estado nutricional (como síndrome da imunodeficiência adquirida ou câncer) ou estar em uso de outro tipo de terapia renal substitutiva. A coleta de dados ocorreu no período de março a dezembro de 2014. Foram avaliados 96 portadores de DRC que realizaram HD em hospitais públicos ou clínicas privadas conveniadas ao SUS em quatro regiões administrativas do Distrito Federal. Do total de pacientes avaliados $35,5 \%$ realizavam HD em hospitais públicos e $64,5 \%$ em clínicas de HD privadas. O estudo foi aprovado pelo Comitê de Ética em Pesquisa da instituição e pelo Comitê de Ética da Fundação de Ensino e Pesquisa em Ciências da Saúde - FEPECS do Distrito Federal. Todos os participantes foram esclarecidos sobre os procedimentos do estudo e assinaram o Termo de Consentimento Livre e Esclarecido.

As informações sociodemográficas foram obtidas por meio de um questionário respondido pelos participantes e incluíram as seguintes informações: raça, procedência, escolaridade, renda e ocupação. Outros dados foram coletados a partir do prontuário médico e incluíram: doença de base, tempo de HD, acesso utilizado na $\mathrm{HD}$ e valores mais recentes de albumina sérica (método de verde de bromocresol) e Kt/V (fórmula do Daugirdas II), parâmetro esse de avaliação da eficiência da diálise. Na fórmula do $\mathrm{Kt} / \mathrm{V}$, o (K) representa a depuração de ureia do dialisador, que é multiplicada pelo tempo de tratamento $(\mathrm{t})$ e dividida pelo volume de distribuição de ureia do paciente $(\mathrm{V})$. A adequação da diálise é fixada pelas diretrizes do National Kidney Foundation Disease Outcomes Quality Initiative (NKF-DOQI), a qual recomenda que se mantenha um valor de Kt/V superior a $1,2^{9}$.

Imediatamente após a sessão de HD foi realizada a avalição antropométrica que incluiu as seguintes medidas: peso e altura (PL-200 kg; Filizola $^{\circledR}$, São Paulo, Brasil), circunferências do braço (CB), cintura (CC) e quadril utilizando fita não elástica e prega cutânea tricipital (PCT) (Plicômetro Científico Classic; Sanny ${ }^{\circledR}$, São Paulo, Brasil). A pesagem do paciente após a sessão de HD faz parte da rotina clínica, uma vez que esta medida representa o peso seco, ou seja, sem excesso de líquidos corporais que, neste caso, foi eliminado através do procedimento de HD. No momento da avaliação, os participantes foram orientados a usar roupas leves e ficarem descalços e centralizados, eretos, com os braços soltos ao longo do corpo e olhar na linha do horizonte. Todas as medidas foram realizadas por um único profissional nutricionista, seguindo protocolo previamente estabelecido ${ }^{10}$.

Para a obtenção dos valores de circunferência muscular do braço (CMB) foi utilizada a fórmula $\mathrm{CMB}(\mathrm{cm})=\mathrm{CB}(\mathrm{cm})-($ PCT em mm x 0,314). Por haver diferenças entre os valores de referência para homens e mulheres, obteve-se valores de adequação percentual para CB, CMB e PCT. Tais valores foram obtidos pela razão entre o valor da medida realizada e o valor de referência da medida (percentil 50). Os resultados foram classificados segundo Frisancho ${ }^{11}$. Para o Índice de Massa Corpórea (IMC) foi utilizada a classificação da Organização Mundial de Saúde ${ }^{12}$ e a de Lipschitz ${ }^{13}$ para participantes com idade igual ou superior a 60 anos. A medida da circunferência da cintura e a relação cintura/quadril (RCQ) foram categorizadas segundo os parâmetros estabelecidos pela OMS (2000), ou seja, $<80 \mathrm{~cm}$ e $<0,85$ (mulheres) e $<94 \mathrm{~cm}$ e $<0,90$ (homens), respectivamente ${ }^{12}$. Valores superiores aos estabelecidos sugerem um aumento no risco de eventos cardiovasculares.

Para a avaliação da desnutrição proteico energética na DRC foram utilizados os critérios propostos pela International Society of Renal $\mathrm{Nu}$ trition and Metabolism ${ }^{14}$ que incluem: albumina sérica $<3,8 \mathrm{~g} / \mathrm{dl}$, IMC $<23 \mathrm{~kg} / \mathrm{m}^{2}$ e $\mathrm{CMB}$ com redução $>10 \%$ em comparação ao percentil 50 da população de referência.

\section{Análise estatística}

Calculou-se médias e desvios-padrão para variáveis quantitativas e percentuais para variáveis qualitativas de características demográficas, clínicas e antropométricas. Com o objetivo de avaliar se houve diferença nos parâmetros avaliados por gênero, foram realizadas comparações das variáveis por meio de teste t-Student ou teste não paramétrico (para variáveis quantitativas) e por teste Qui-quadrado ou Exato de Fisher (para variáveis qualitativas). Coeficientes de correlação de Pearson foram estimados entre variáveis quantitativas. Resultados de testes com p-valor inferior 
a 0,05 foram consideradas estatisticamente significantes. Todas as análises estatísticas foram implementadas no software R (versão 3.2.0).

\section{Resultados}

A hipertensão arterial, isoladamente, foi a doença de base mais prevalente, seguida pelo diabetes. Apenas $14 \%$ dos pacientes avaliados eram diabéticos e hipertensos. O perfil mais comum da amostra foi de indivíduos pardos, oriundos da região Nordeste, com baixo nível de escolaridade e renda mensal de até dois salários mínimos. As demais características sociodemográficas dos participantes são apresentadas na Tabela 1 .

A média de tempo em HD foi de 3 anos e 11 meses (mínimo: 3 meses; máximo: 21 anos), sendo a fistula arteriovenosa o principal tipo de acesso utilizado para o procedimento ( $83 \%$ vs. $17 \%$ cateter). Quanto ao Kt/V, dos 96 pacientes avaliados somente $58(60 \%)$ dispunham de algum valor desta variável registrado em prontuário no período da coleta de dados (54 \pm 15 anos; $57 \%$ masculino; $\mathrm{Kt} / \mathrm{V}=1,3 \pm 0,3)$. Os resultados encontrados acima do limite preconizado se justificam em função de valores superiores em mulheres quando comparado aos homens $(1,5 \pm 0,3$ vs. 1,2 $\pm 0,2$ em homens; $P=0,004)$. No entanto, $35 \%$ dos pacientes desta sub-amostra apresentaram valor de $\mathrm{Kt} / \mathrm{V}$ inferior ou igual a 1,2 ( $\mathrm{n}=18$; $53 \pm 11$ anos; $72 \%$ masculino).

A Tabela 2 apresenta os resultados das variáveis relacionadas ao estado nutricional. No que se refere à avaliação antropométrica, observou-se que entre os participantes adultos, a maior parte $(53 \%)$ enquadrou-se nos padrões de eutrofia estabelecidos pela $\mathrm{OMS}^{12}$, seguidos por participantes com sobrepeso/obesidade e com magreza. Não foram observadas diferenças na média ou na distribuição percentual dos grupos de IMC entre os gêneros. Resultados semelhantes foram encontrados quando os pacientes idosos foram analisados separadamente segundo a classificação para a faixa etária igual ou superior a 60 anos $(\mathrm{n}=36 ; 69$ \pm 5 anos; $50 \%$ masculino $)^{13}$.

Setenta e três por cento dos participantes apresentou algum grau de desnutrição (leve, moderada ou grave), segundo a adequação de PCT. O mesmo padrão de resposta foi observado para $\mathrm{CB}$ (70,9\% de desnutrição) e, em menor grau, para CMB (41\% de desnutrição). Para as medidas CB e CMB, o tipo de desnutrição mais frequente foi a leve, seguido de eutrofia e sobrepeso/obesidade, respectivamente. Já para PCT, a desnutrição grave foi mais frequente. Não foram observadas diferenças estatisticamente significantes entre os gêneros no percentual de adequação obtido para estas medidas. No entanto, foi demonstrado que o percentual de homens com valores adequados de circunferência de cintura e relação cintura/ quadril foi superior ao encontrado em mulheres $(\mathrm{P}<0,05)$.

Os valores de albumina sérica encontrados foram, em média, ligeiramente superiores ao ponto de corte proposto $(3,85 \pm 0,43 \mathrm{~g} / \mathrm{dl})$, sendo maiores em homens $(3,94 \pm 0,40 \mathrm{~g} / \mathrm{dl})$ do que em mulheres $(3,72 \pm 0,44 \mathrm{~g} / \mathrm{dl} ; \mathrm{P}=0,01)$. Apesar deste achado, não houve diferença entre o percentual de mulheres e homens que apresentaram um valor de albumina abaixo de $3,8 \mathrm{~g} / \mathrm{dl}$, como parte dos critérios de desnutrição sugeridos pela Sociedade Internacional de Nutrição Renal e Metabolismo $(\text { ISRNM })^{14}$. De acordo com os resultados apresentados na tabela 2, pode-se observar que homens apresentaram IMC $<23 \mathrm{~kg} / \mathrm{m}^{2}$ em maior proporção em comparação às mulheres. No entanto, não houve diferença entre os gêneros para o critério referente à CMB.

Ao se considerar conjuntamente os valores de albumina sérica, IMC, e CMB, observou-se que $14,6 \%$ dos participantes foram classificados como desnutridos (60 \pm 12 anos; $57 \%$ masculino; $69 \%$ clínicas privadas). A Figura 1 ilustra a distribuição dos participantes quanto à adequação aos três critérios de desnutrição na IRC. Do total de indivíduos avaliados $21,9 \%$ atenderam a dois critérios de desnutrição; $30,2 \%$ atenderam a um critério e 33,3\% não atenderam a nenhum dos critérios, ou seja, apresentaram valores de IMC, CMB e albumina sérica acima dos limites para caracterização de desnutrição (55 \pm 14 anos; 53\% masculino; $57 \%$ clínicas privadas). Ao considerar os pacientes com 0 a 2 critérios positivos para desnutrição, ou seja, não desnutridos, observou-se um padrão de eutrofia para IMC e adequação de CMB e desnutrição leve para adequação CB e PCT em comparação ao grupo desnutrido $(\mathrm{P}<0,01)$.

\section{Discussão}

O presente estudo investigou o estado nutricional de portadores de DRC em tratamento hemodialítico pelo SUS no DF. Do total de indivíduos avaliados, $14,6 \%$ foram classificados como desnutridos, $33,3 \%$ apresentaram estado nutricional adequado e $52,1 \%$ apresentaram ao menos uma variável relacionada à desnutrição. Nesse grupo, dentre os parâmetros analisados, o IMC abaixo do recomendado $\left(<23 \mathrm{~kg} / \mathrm{m}^{2}\right)$ foi o mais prevalente. 
Tabela 1. Características gerais, sócio-demográficas e relacionadas à doença renal em portadores de insuficiência renal crônica em hemodiálise.

\begin{tabular}{|c|c|c|c|}
\hline Variável & $\begin{array}{l}\text { Todos } \\
(\mathrm{n}=96)\end{array}$ & $\begin{array}{c}\text { Feminino }^{*} \\
(n=41)\end{array}$ & $\begin{array}{c}\text { Masculino* }^{*} \\
(\mathrm{n}=55)\end{array}$ \\
\hline \multicolumn{4}{|l|}{ Clínicas (Tipo/Localidade),\% } \\
\hline Público/Asa Norte & 16,7 & 17,1 & 14,5 \\
\hline Público/Taguatinga & 18,8 & 24,4 & 16,4 \\
\hline Privado/Asa Sul & 8,3 & 4,9 & 23,6 \\
\hline Privado/Ceilândia & 22,9 & 22,0 & 34,6 \\
\hline Privado/Samambaia & 33,3 & 31,6 & 10,9 \\
\hline \multicolumn{4}{|l|}{ Doença de Base, $\%$} \\
\hline HAS & 43,7 & 43,8 & 43,6 \\
\hline Diabetes & 27,1 & 29,3 & 25,5 \\
\hline Glomerulonefrite & 9,4 & 4,9 & 12,7 \\
\hline Outras causas & 19,8 & 22,0 & 18,2 \\
\hline Idade, média (D.P.) & $54,2(15,0)$ & $54,4(16,1)$ & $54,1(14,2)$ \\
\hline$\geq 60$ anos, $\%$ & 37,5 & 43,9 & 32,7 \\
\hline \multicolumn{4}{|l|}{ Raça, \% } \\
\hline Parda & 54,2 & 48,9 & 58,2 \\
\hline Branca & 20,8 & 26,8 & 10,9 \\
\hline Negra & 17,7 & 19,5 & 21,8 \\
\hline Indígena & 4,2 & 2,4 & 5,5 \\
\hline Não informado & 3,1 & 2,4 & 3,6 \\
\hline \multicolumn{4}{|l|}{ Região de Origem, \% } \\
\hline Nordeste & 54,2 & 61,0 & 49,1 \\
\hline Centro-Oeste & 31,2 & 29,2 & 32,7 \\
\hline Sudeste & 14,6 & 9,8 & 18,2 \\
\hline \multicolumn{4}{|l|}{ Escolaridade, \% } \\
\hline Analfabeto & 13,5 & 14,6 & 12,7 \\
\hline Lê e escreve & 1,0 & 2,4 & 0,0 \\
\hline Fundamental incompleto & 35,5 & 36,7 & 34,5 \\
\hline Fundamental completo & 12,5 & 14,6 & 10,9 \\
\hline Médio incompleto & 13,5 & 12,2 & 14,5 \\
\hline Médio completo & 16,7 & 17,1 & 16,4 \\
\hline Superior incompleto & 4,2 & 2,4 & 5,5 \\
\hline Superior completo & 3,1 & 0,0 & 5,5 \\
\hline \multicolumn{4}{|l|}{ Renda, \% } \\
\hline$\leq 2$ salários mínimos & 86,4 & 95,2 & 80,0 \\
\hline 3-4 salários mínimos & 6,3 & 2,4 & 9,1 \\
\hline$\geq 5$ salários mínimos & 7,3 & 2,4 & 10,9 \\
\hline
\end{tabular}

Notas: ${ }^{\star}$ As porcentagens das terceira e quarta colunas foram calculadas com base nos totais de participantes dos sexos feminino e masculino, respectivamente. Não houve diferença estatisticamente significante entre os gêneros para nenhuma das variáveis apresentadas nesta tabela.

HAS - Hipertensão Arterial Sistêmica.

A DRC é uma enfermidade complexa, com impacto significante na qualidade de vida, longevidade, uso de recursos médicos e gastos em saúde pública ${ }^{15-17}$. O portador de DRC experimenta uma drástica mudança em sua vida, passando a conviver com muitas limitações que incluem tratamento hemodialítico doloroso, dietoterapia rígida, mudanças no cotidiano familiar, profissional e social e pensamento na morte mais frequente, $o$ que leva o indivíduo a ter uma percepção negativa sobre sua saúde ${ }^{18,19}$. Estudos que envolvam análise do perfil da DRC no Brasil são de grande importância, de forma que seja possível reunir informações com base na nossa população e no sistema de saúde local, para planejar uma abordagem correta da DRC em termos de políticas públicas ${ }^{20}$.

No Brasil, a Política Nacional de Atenção ao Portador de Doença Renal ${ }^{21}$ foi instituída em 2004 tendo como uma de suas principais metas definir critérios técnicos mínimos para o funcionamento e avaliação dos serviços públicos e privados que realizam diálise, bem como os me- 
Tabela 2. Caracterização do estado nutricional de portadores de insuficiência renal crônica em hemodiálise.

\begin{tabular}{|c|c|c|c|c|}
\hline Variável & $\begin{array}{c}\text { Todos } \\
(\mathrm{n}=96)\end{array}$ & $\begin{array}{c}\text { Feminino } \\
(\mathrm{n}=41)\end{array}$ & $\begin{array}{c}\text { Masculino } \\
(\mathrm{n}=55)\end{array}$ & p-valor \\
\hline Albumina (g/dl), mediana (D.I.) & $3,9(0,5)$ & $3,8(0,5)$ & $4,0(0,5)$ & $0,006^{\mathrm{w}}$ \\
\hline \multicolumn{5}{|l|}{ Critérios de desnutrição da ISRNM } \\
\hline Albumina $<3,8 \mathrm{~g} / \mathrm{dl}, \%$ & 33,3 & 43,9 & 25,5 & $0,09^{\mathrm{q}}$ \\
\hline $\mathrm{IMC}<23, \%$ & 42,7 & 26,8 & 52,7 & $0,02 \mathrm{q}$ \\
\hline CMB limite negativo, $\%$ & 41,7 & 43,9 & 41,8 & $0,99 \mathrm{q}$ \\
\hline Atendem aos 3 critérios, $\%$ & 14,6 & 14,6 & 14,5 & $0,99^{q}$ \\
\hline IMC $\left(\mathrm{kg} / \mathrm{m}^{2}\right)$, média (D.P.) & $24,4(4,6)$ & $24,0(4,8)$ & $24,7(4,5)$ & $0,44^{\mathrm{t}}$ \\
\hline IMC categorizado OMS, \% & & & & $0,67^{\mathrm{f}}$ \\
\hline Magreza & 6,3 & 9,8 & 3,6 & \\
\hline Eutrofia & 53,1 & 53,6 & 52,8 & \\
\hline Sobrepeso & 28,1 & 24,4 & 30,9 & \\
\hline Obesidade & 12,5 & 12,2 & 12,7 & \\
\hline IMC categorizado por idade ${ }^{\star}, \%$ & & & & $0,75^{\mathrm{q}}$ \\
\hline Magreza & 13,5 & 14,6 & 12,7 & \\
\hline Eutrofia & 48,0 & 51,3 & 45,5 & \\
\hline Sobrepeso/Obesidade & 38,5 & 34,1 & 41,8 & \\
\hline Circunferência de Cintura Adequada, \% & 53,1 & 35,9 & 67,3 & $0,005^{\mathrm{q}}$ \\
\hline RCQ Adequada, \% & 42,7 & 23,1 & 58,2 & $0,002^{\mathrm{q}}$ \\
\hline Adequação de PCT, mediana (D.I.*) & $76,9(38,75)$ & $75,0(51,7)$ & $79,2(26,1)$ & $0,66^{\mathrm{w}}$ \\
\hline Adequação de PCT, \% & & & & $0,20^{\mathrm{q}}$ \\
\hline Desnutrição Grave (<70\%) & 41,7 & 46,4 & 38,2 & \\
\hline Desnutrição Moderada (70 a 80\%) & 13,5 & 14,6 & 12,7 & \\
\hline Desnutrição Leve (80 a 90\%) & 17,7 & 7,3 & 25,5 & \\
\hline Eutrofia (90 a 110\%) & 12,5 & 12,2 & 12,7 & \\
\hline Sobrepeso (110 a $120 \%)$ & 0,0 & 0,0 & 0,0 & \\
\hline Obesidade $(\geq 120 \%)$ & 14,6 & 19,5 & 10,9 & \\
\hline Adequação de CB, média (D.P.) & $83,9(11,5)$ & $86,7(13,9)$ & $83,3(9,3)$ & $0,15^{\mathrm{t}}$ \\
\hline Adequação de CB, \% & & & & $0,09^{\mathrm{f}}$ \\
\hline Desnutrição Grave $(<70 \%)$ & 9,4 & 14,6 & 5,5 & \\
\hline Desnutrição Moderada (70 a 80\%) & 21,9 & 12,2 & 29,1 & \\
\hline Desnutrição Leve (80 a 90\%) & 39,6 & 36,7 & 41,8 & \\
\hline Eutrofia (90 a $110 \%)$ & 28,1 & 34,1 & 23,6 & \\
\hline Sobrepeso (110 a 120\%) & 1,0 & 2,4 & 0,0 & \\
\hline Obesidade $(\geq 120 \%)$ & 0,0 & 0,0 & 0,0 & \\
\hline Adequação de CMB, média (D.P.) & $93,7(14,0)$ & $96,2(12,5)$ & $91,8(14,8)$ & $0,13^{\mathrm{t}}$ \\
\hline Adequação de CMB, \% & & & & $0,10^{\mathrm{q}}$ \\
\hline Desnutrição Grave $(<70 \%)$ & 2,1 & 0,0 & 3,6 & \\
\hline Desnutrição Moderada (70 a 80\%) & 14,6 & 12,2 & 16,4 & \\
\hline Desnutrição Leve (80 a 90\%) & 25,0 & 14,6 & 32,7 & \\
\hline Eutrofia (90 a 100\%) & 27,1 & 34,1 & 21,8 & \\
\hline Sobrepeso/Obesidade $(\geq 100 \%)$ & 31,2 & 39,1 & 25,5 & \\
\hline
\end{tabular}

Notas: IMC - Índice de Massa Corpórea, RCQ - razão cintura-quadril, PCT - Prega Cutânea Triciptal, CB - Circunferência do Braço, CMB -Circunferência Muscular do Braço. Classificação IMC (OMS): magreza (IMC $\left.<18,5 \mathrm{~kg} / \mathrm{m}^{2}\right)$; eutrofia $(18,5 \leq \mathrm{IMC}<$ $\left.25 \mathrm{~kg} / \mathrm{m}^{2}\right)$; sobrepeso. $\left(25 \leq \mathrm{IMC}<30 \mathrm{~kg} / \mathrm{m}^{2}\right)$; obesidade (IMC $\left.\geq 30 \mathrm{~kg} / \mathrm{m}^{2}\right)$. ${ }^{*}$ Classificação para $\geq 60$ anos: magreza (IMC $<22 \mathrm{~kg} /$ $\left.\mathrm{m}^{2}\right)$, eutrofia $\left(22 \leq \mathrm{IMC}<27 \mathrm{~kg} / \mathrm{m}^{2}\right)$, sobrepeso/obesidade $\left(\mathrm{IMC} \geq 27 \mathrm{~kg} / \mathrm{m}^{2}\right)$. ${ }^{*}$ D.I. representa desvio interquartílico, calculado pela diferença entre o terceiro e o primeiro quartis. " $\mathrm{p}$-valor referente a teste não-paramétrico de Wilcoxon-Mann-Whitney para comparação por sexo. ${ }^{9}$ p-valor referente a teste Qui-quadrado para comparação por sexo. ${ }^{\mathrm{f}} \mathrm{p}$-valor referente a teste exato de Fisher para comparação por sexo. ${ }^{t} \mathrm{p}$-valor referente a teste t-Student para comparação por sexo.

canismos de sua monitoração com vistas a diminuir os riscos aos quais fica exposto o portador de doença renal. No âmbito do SUS, as Diretrizes Clínicas para o Cuidado ao Paciente com DRC 22 estabelecem a necessidade de acompanhamento por uma equipe multiprofissional nas Unidades Básicas de Saúde e, nos casos que requerem, nas unidades de atenção especializada em DRC para orientações e educação, incluindo aconselhamento e suporte sobre mudança do estilo de 


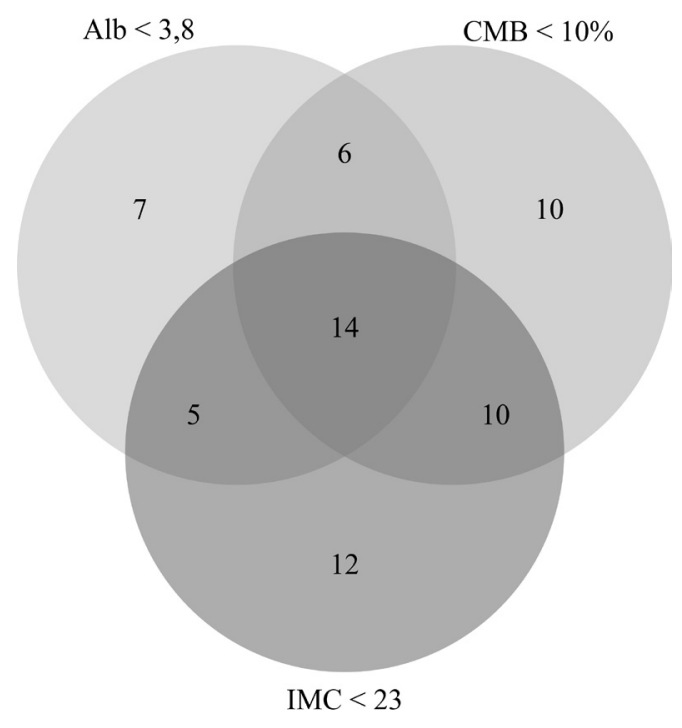

Figura 1. Diagrama de Venn para o número de participantes que atendem a pelo menos um dos critérios do IRNS que incluem albumina sérica $<$ 3,8 g/dl (Alb < 3,8; n=32), CMB com redução > $10 \%$ em comparação ao percentil 50 da população de referência $(\mathrm{CMB}<10 \%$; $=40)$ e $\mathrm{IMC}<23 \mathrm{~kg} /$ $\mathrm{m}^{2}(\mathrm{n}=41)$. Os 33 participantes que não atenderam a nenhum dos 3 critérios não estão incluídos nesse diagrama.

vida, avaliação nutricional, orientação sobre o auto-cuidado, entre outros.

O número absoluto de pacientes em diálise tem aumentado 3\% ao ano nos últimos três anos. A HD se mantém como o tipo de tratamento mais frequente, com maior prevalência em homens e a hipertensão arterial, como o principal diagnóstico primário, seguido de diabetes ${ }^{2,23,24}$. O perfil identificado no presente estudo, em que 57\% dos avaliados eram homens, 45,5\% hipertensos, além de outras características que incluíram baixo nível de escolaridade e renda inferior a dois salários mínimos foi semelhante ao encontrado na literatura $^{2,23}$. Todos os avaliados eram assistidos pelo SUS, seja em estabelecimentos públicos ou privados conveniados. Zambonato et al. ${ }^{25}$ observaram uma associação significativa entre o aumento na mortalidade e marcadores de menor status socioeconômico como menor escolaridade, classe econômica C, D ou E e ausência de plano de saúde privado em uma região no Sul do Brasil. Este fato se deve à falta de tratamento das doenças de base (hipertensão e diabetes) e/ou encaminhamento tardio ao nefrologista para inicio do tratamen- to ${ }^{17,26}$. No entanto, a análise de sobrevida em uma coorte de 3.082 pacientes em HD demonstrou uma taxa de mortalidade relativamente baixa, justificada pelo fato da população ser mais jovem e com prevalência de diabetes mais baixa do que aquela descrita em países desenvolvidos ${ }^{24}$.

Numerosos estudos vêm demonstrando a correlação entre a dose de HD e a morbimortalidade de portadores de DRC ${ }^{27,28}$. Nesse sentido, para avaliar se estes indivíduos estão recebendo o tratamento adequado é necessário mensurar a dose de HD que está sendo administrada além de avaliar sinais clínicos e sintomas associados. O Kt/V (depuração de uréia do dialisador $\mathrm{x}$ tempo de tratamento/ volume de distribuição de ureia do paciente) representa um dado de grande importância na avaliação da qualidade e adequação do tratamento dialítico ${ }^{29}$. Parâmetros internacionais foram estabelecidos para esta variável, em que os valores mínimos de Kt/V recomendados para três sessões por semana devem ser maiores que $1,2{ }^{9}$. Atualmente há máquinas de diálise que oferecem um monitor on -line da eficiência da diálise e mostram o Kt/V em tempo real na tela. Embora a média dos resultados de Kt/V do grupo avaliado no presente estudo estivesse ligeiramente acima dos parâmetros de referência, apenas $60 \%$ dos indivíduos dispunham de algum valor desta variável medida nos meses anteriores, contrariando diretrizes nacionais e internacionais que recomendam o controle da dose de diálise ao menos uma vez por mês.

Assim como a dose inadequada de $\mathrm{HD}$, os níveis de albumina sérica baixos também estão correlacionados a índices de morbimortalidade aumentados nesses pacientes ${ }^{30}$. Em nosso estudo, $67 \%$ dos indivíduos avaliados apresentaram valores de albumina adequados. Dados do $1^{\circ}$ Censo do Estado Nutricional de Pacientes em Hemodiálise realizado pela Sociedade Brasileira de $\mathrm{Ne}$ frologia também mostraram valores adequados de Kt/V e albumina sérica em estabelecimentos de HD na região Centro-Oeste, acompanhando a tendência nacional $(77,9 \%$ dos pacientes com valores superiores a 1,2 e $88,4 \%$ com resultados superiores a $3,8 \mathrm{mg} / \mathrm{dl}$, respectivamente $)^{8}$.

A albumina sérica é o marcador bioquímico mais utilizado para avaliação da desnutrição proteico-calórica na $\mathrm{HD}^{14,30,31}$. Isso se deve ao fato de a desnutrição ser um achado frequente nestes indivíduos, o que torna seu diagnóstico precoce uma ferramenta importante na redução das taxas de morbi-mortalidade. A importância da nutrição no cuidado com a saúde renal ocorre desde o contexto das medidas preventivas, porém, uma vez instalada a patologia renal a nutrição desempenha 
um papel central na avaliação e no tratamento dessa doença ${ }^{32}$. A DRC, seja na fase pré-dialítica ou dialítica, impõe desafios clínicos diretamente ligados ao estado nutricional. No entanto, a identificação da desnutrição nos pacientes em diálise em casos menos exuberantes é considerada um desafio em comparação a estágios mais avançados da DRC, podendo surgir controvérsias relacionadas aos parâmetros utilizados para o diagnóstico ${ }^{33}$. O melhor método de identificação da desnutrição energético-protéica em pacientes em HD ainda se mantém em debate ${ }^{30}$. Apesar de todo o esforço em se aproximar do diagnóstico nutricional preciso, ainda falta uma normatização dos parâmetros nutricionais a serem aplicados neste caso.

Um comitê internacional (International Society of Renal Nutrition and Metabolism) propôs um conjunto de indicadores nutricionais que devem ser usados para identificar o paciente com DRC portador de desnutrição, o qual abrange parâmetros bioquímicos, massa corporal, massa muscular e consumo alimentar ${ }^{14}$. Dentre os indicadores bioquímicos, a albumina sérica é a variável que apresenta maior poder em prever mortalidade nos estudos epidemiológicos de pacientes em diálise. Quanto à massa corporal, uma redução no IMC sugere um quadro de desnutrição em indivíduos com DRC. Por fim, uma redução na massa muscular parece ser o critério mais válido da presença de desnutrição, podendo ser avaliada por diferentes métodos, incluindo a antropometria, que é de baixo custo, mas exige profissional treinado para sua execução.

Nos resultados do presente estudo observou-se que dentre os pacientes avaliados, 42,7\% apresentaram IMC abaixo da meta; em 41,7\% os valores de circunferência muscular do braço, um indicador de massa muscular, estavam diminuídos e 33,3\% apresentaram albumina sérica inferior ao recomendado. Ao analisar as variáveis conjuntamente, $14,6 \%$ dos portadores de DRC em tratamento hemodialítico foram classificados como desnutridos. Os graus de desnutrição na DRC relatados na literatura variam amplamente e mesmo os indivíduos com sobrepeso ou obesidade apresentam risco de desnutrição proteica apesar da ingesta calórica aumentada ${ }^{34}$. Vegine et al..$^{30}$ encontraram valores de IMC e CMB adequados em um grupo de indivíduos com DRC em HD. No entanto, segundo a PCT, o grupo foi classificado com desnutrição moderada. Quando os critérios da ISRNM foram aplicados, apenas 13,3\% pacientes foram classificados como desnutridos e $40,6 \%$ apresentaram sobrepeso ou obesidade. No Inquérito Brasileiro de Diálise Crônica ${ }^{2}$ a desnutrição foi diagnosticada em $10 \%$ pacientes, enquanto $37 \%$ dos avaliados apresentaram sobrepeso/obesidade. Esse percentual é inferior ao reportado na população geral brasileira (53\%) em $2014^{35}$ e também é inferior à encontrada na população em diálise nos EUA, onde o IMC médio dos pacientes tem aumentado nos últimos anos ${ }^{36}$.

Nossos resultados se assemelham aos obtidos no Censo do Estado Nutricional de Pacientes em Hemodiálise $^{8}$, onde os pacientes, em sua maioria, eram eutróficos segundo o IMC e apresentavam valores de Kt/V e albumina adequados. Os dados do Censo apontaram ainda que, em comparação aos pacientes de convênios médicos, aqueles assistidos pelo SUS apresentaram perfil semelhante para estas variáveis (Eutrofia/IMC: $49 \%$ vs $56 \%$ SUS; Albumina $\geq 3,5: 86,6 \%$ vs $87,9 \%$ SUS; Kt/V $\geq 1,2: 75,5 \%$ vs $80 \%$ SUS). No entanto, tais dados devem ser avaliados com cautela tendo em vista o alto nível de não -resposta observado (dados de 50\% dos pacientes de 12\% das Unidades Renais Cadastradas).

Em todas as cinco clínicas envolvidas no estudo, sendo três de administração privada e conveniadas ao SUS, a maior proporção observada foi de portadores de IRC com ao menos uma variável relacionada ao estado nutricional alterada (IMC, albumina ou $\mathrm{CMB}$ ), seguida de indivíduos com bom estado nutricional e finalmente indivíduos desnutridos, ou seja, com as três variáveis alteradas. A forte demanda dos doentes renais crônicos por tratamento e pelas características da diálise (procedimento relativamente simples), fez com que o tratamento dialítico começasse a ganhar espaço, com o credenciamento de unidades de hemodiálise pelo então Instituto Nacional de Assistência Médica da Previdência Social (INAMPS) que a partir de meados da década de 70 se espalharam por todo o país. Ao permitir esse rápido crescimento, o INAMPS despertou uma massa de interesses (a atraente remuneração paga pelos procedimentos dialíticos garantiu o interesse dos prestadores de serviços em expandir a oferta), que desde o início da década de 1980 têm conseguido preservar os níveis de remuneração da atividade e com isso garantir sua contínua expansão $0^{37,38}$.

No que se refere às diferenças encontradas entre os gêneros, observamos que os homens apresentaram valores superiores de albumina sérica, embora o percentual daqueles nos quais foi identificado um IMC abaixo do recomendado $\left(<23 \mathrm{~kg} / \mathrm{m}^{2}\right)$ tenha sido maior em comparação às mulheres. Provavelmente, em função do IMC reduzido, foi observado maior numero de indivíduos do sexo masculino com valores de circunferência de cintura e relação cintura/qua- 
dril adequados. O gênero tem sido considerado um fator importante na progressão da doença renal ${ }^{39}$. As mulheres tendem a progredir mais lentamente para um estágio avançado de doença renal em comparação aos homens, independente da etiologia ${ }^{40}$. Várias hipóteses são sugeridas para explicar este achado, dentre elas, a diferença no impacto que o estilo de vida e fatores de risco tradicionais exercem sobre mulheres e homens com $\mathrm{DRC}^{41}$. Estudos anteriores demonstraram que homens com DRC apresentam hábitos alimentares piores e aderem menos às restrições dietéticas como parte do tratamento da doença ${ }^{42}$. Outro estudo demonstrou que fatores de risco modificáveis, incluindo o IMC, contribuem para a progressão acelerada da doença em homens ${ }^{43}$. Em resumo, parece haver diferença entre gêneros em relação às variáveis preditoras de declínio da função renal, mas não está claro se estas variáveis podem ser modificadas pelo fator gênero ${ }^{39}$.

$\mathrm{O}$ estudo apresenta limitações que incluem a utilização de uma amostra por conveniência, já que apenas parte das clínicas que aceitaram participar do estudo foram incluídas. A análise do perfil bioquímico dos voluntários se restringiu às dosagens de albumina sérica. Além disso, não foi realizado registro do consumo alimentar como parte da avaliação do estado nutricional.

\section{Conclusão}

A análise do estado nutricional segundo os critérios propostos pela International Society of Renal Nutrition and Metabolism em portadores de DRC em tratamento hemodialítico pelo SUS no DF demonstrou que $14,6 \%$ dos indivíduos avaliados apresentavam desnutrição. Dentre os parâmetros utilizados para avaliação do estado nutricional, o IMC abaixo do recomendado foi o critério mais prevalente, tendo sido observado em 42,7\% da amostra.

Os resultados encontrados reforçam a importância da avaliação nutricional no contexto do tratamento de renais crônicos em hemodiálise. Em função da diversidade de variáveis e da dificuldade no estabelecimento de um procedimento padrão para este grupo, é importante o conhecimento por parte do profissional de saúde sobre quais parâmetros usados para avaliação do estado nutricional são capazes de identificar melhor a presença de desnutrição no paciente com DRC.

\section{Colaboradores}

K Bousquet-Santos contribuiu para o desenho do estudo, supervisão da coleta de dados, análise estatística e redação do manuscrito. LG Costa contribuiu para o desenho do estudo, coleta de dados e parte da análise estatística. JML Andrade contribuiu para a análise estatística e redação do manuscrito.

\section{Agradecimentos}

Às clínicas participantes, as quais concordaram em participar do estudo e permitiram o acesso dos pesquisadores durante a fase de coleta de dados.

À aluna Mariana Idnês de O. Mendes pela colaboração na fase de coleta dos dados.

Aos pacientes, pela gentileza com que nos receberam e sem os quais esse estudo não seria viável. 


\section{Referências}

1. Marinho F, Passos VMA, França EB. Novo século, novos desafios: mudança no perfil da carga de doença no Brasil de 1990 a 2010. Epidemiol Serv Saúde 2016; 25(4):713-724.

2. Sesso RC, Lopes AA, Thomé FS, Lugon JR, Martins CT. Brazilian chronic dialysis census 2014. J Bras Nefrol 2016; 38(1):54-61.

3. Pecoits-Filho R, Rosa-Diez G, Gonzalez-Bedat M, Marinovich S, Fernandez S, Lugon J, Poblete-Badal H, Elgueta-Miranda S, Gomez R, Cerdas-Calderon M, Almaguer-Lopez M, Freire N, Leiva-Merino R, Rodriguez G, Luna-Guerra J, Bochicchio T, Garcia-Garcia G, Cano N, Iron N, Cuero C, Cuevas D, Tapia C, Cangiano J, Rodriguez S, Gonzalez H, Duro-Garcia V. Renal replacement therapy in CKD: an update from the Latin American Registry of Dialysis and Transplantation. $J$ Bras Nefrol 2015; 37(1):9-13.

4. Oliveira CMC, Kubrusly M, Mota RS, Silva CAB, Oliveira VN. Desnutrição na insuficiência renal crônica: qual o melhor método diagnóstico na prática clínica? J Bras Nefrol 2010; 32(1):57-70.

5. Zha Y, Qian Q. Protein Nutrition and Malnutrition in CKD and ESRD. Nutrients 2017; 9(3):208.

6. Vegine PM, Fernandes ACP, Torres MRSG, Silva MIB, Avesani CM. Avaliação de métodos para identificar desnutrição energético-protéica de pacientes em hemodiálise. J Bras Nefrol 2011;33(1):55-61.

7. Freitas ATVS, Vaz IMF, Ferraz SF, Peixoto MRG, Campos MIVM. Prevalência de desnutrição e fatores associados em pacientes em hemodiálise. Rev Nutr 2014; 27(3):357-366.

8. Biavo BMM, Branco CT, Cunha LM, Araujo ML, Ribeiro MMC, Sachs A, Uezima CBB, Draibe AS, Rodrigues CIS, Barros EJG. Aspectos nutricionais e epidemiológicos de pacientes com doença renal crônica submetidos a tratamento hemodialítico no Brasil, 2010. J Bras Nefrol 2012; 34(3):206-402.

9. National Kidney Foundation. KDOQI clinical practice guideline for hemodialysis adequacy: 2015 update. Am J Kidney Dis 2015; 66(5):884-930.

10. Ross WD, Marfell-Jones M. Kinanthropometry. In: MacDougall JD, Wenger HA, Green HJ, organizadores. Physiological testing of the high-performance athlete. Champaign: Human Kinetics; 1991. p. 223-308.

11. Frisancho AR. New norms of upper limb fat and muscle areas for assessment of nutritional status. Am J Clin Nutr 1981; 34(11):2540-2545.

12. World Health Organization (WHO). Obesity: preventing and managing the global epidemic. Report of a WHO consultation. World Health Organ Tech Rep Ser 2000; 894:1-253.

13. Lipschitz D. Screening for nutritional status in the elderly. Prim Care 1994; 21(1):55-67.

14. Fouque D, Kalantar-Zadeh K, Kopple J, Cano N, Chauveau P, Cuppari L, Franch H, Guarnieri G, Ikizler TA, Kaysen G, Lindholm B, Massy Z, Mitch W, Pineda E, Stenvinkel P, Treviño-Becerra A, Wanner C. A proposed nomenclature and diagnostic criteria for protein-energy wasting in acute and chronic kidney disease. Kidney Int 2008; 73(4):391-398.
15. Szuck P, Fuhr LM, Garcia MF, Silva AT, Wazlawik E. Associação entre indicadores nutricionais e risco de hospitalização em pacientes em hemodiálise. Rev Nutr 2016; 29(3):317-327.

16. Alvares J, Almeida AM, Szuster DA, Gomes IC, Andrade EI, Acurcio FA, Cherchiglia ML. Fatores associados à qualidade de vida de pacientes em terapia renal substitutiva no Brasil. Cien Saude Colet 2013; 18(7):1903-1910.

17. Pena PF, Silva Júnior AG, Oliveira PT, Moreira GA, Libório AB. Cuidado ao paciente com Doença Renal Crônica no nível primário: pensando a integralidade e o matriciamento. Cien Saude Colet 2012; 17(11):31353144.

18. Moreira TR, Giatti L, Cesar CC, Andrade EIG, Acurcio FA, Cherchiglia ML. Autoavaliação de saúde por pacientes em hemodiálise no Sistema Único de Saúde. Rev Saude Publica 2016; 50(10):1-11.

19. Cruz VFES, Tagliamento G, Wanderbroocke AC. A manutenção da vida laboral por doentes renais crônicos em tratamento de hemodiálise: uma análise dos significados do trabalho. Saúde Soc 2016; 25(4):1050-1063.

20. Matos JPS, Lugon JR. Time to ascertain the extent of chronic kidney disease in Brazil. J Bras Nefrol 2014; 36(3):267-268.

21. Brasil. Portaria no 1.168 , de 15 de junho de 2004. Institui a política nacional de atenção ao portador de doença renal, a ser implantada em todas as unidades federadas, respeitadas as competências das três esferas de gestão. Diário Oficial da União 2004; 15 jun.

22. Brasil. Ministério da Saúde (MS). Diretrizes clínicas para o cuidado ao paciente com doença renal crônica DRC no Sistema Único de Saúde. Brasília: MS; 2014.

23. Sesso RC, Lopes AA, Thomé FS, Lugon JR, Watanabe Y, Santos DR. Report of the brazilian chronic dialysis census 2012. J Bras Nefrol 2014; 36(1):48-53.

24. Matos J, Almeida J, Guinsburg A, Marelli C, Barra ABL, Vasconcellos MS, José D’Almeida Filho E, Hoette M, Ruzany F, Lugon JR. Avaliação da sobrevida de cinco anos em hemodiálise no Brasil. J Bras Nefrol 2011; 33(4):436-441.

25. Zambonato TK, Thomé FS, Gonçalves LFS. Perfil socioeconômico dos pacientes com doença renal crônica em diálise na região noroeste do Rio Grande do Sul. J Bras Nefrol 2008; 30(3):192-199.

26. Obialo CI, Ofili EO, Quarshie A, Martin PC. Ultralate referral and presentation for renal replacement therapy: socioeconomic implications. Am J Kidney Dis 2005; 46(5):881-886.

27. Đurić PS, Popović J, Janković A, Tošić J, Dimković N. Parameters of hemodialysis adequacy and patients' survival depending on treatment modalities. Med Pregl 2015; 68(7-8):251-257.

28. Theofilou P, Togas C, Vasilopoulou C, Minos C, Zyga $\mathrm{S}$, Tzitzikos G. The impact of Kt/V urea-based dialysis adequacy on quality of life and adherence in haemodialysis patients: a cross-sectional study in Greece. Heal Psychol Res 2015; 3(1060):7-8.

29. Breitsameter G, Figueiredo AE, Kochhann DS. Cálculo de Kt/V em hemodiálise: comparação entre fórmulas. J Bras Nefrol 2012; 34(1):22-26. 
30. Vegine PM, Fernandes ACP, Torres MRSG, Silva MIB, Avesani CM. Avaliação de métodos para identificar desnutrição energético-protéica de pacientes em hemodiálise. J Bras Nefrol 2011; 33(1):55-61.

31. Cuppari L, Kamimura A. Nutritional evaluation in chronic kidney disease: challenges in clinical practice. Nefrologia 2009; 31(1):28-35.

32. Obi Y, Qader H, Kovesdy CP, Kalantar-Zadeh K. Latest consensus and update on protein-energy wasting in chronic kidney disease. Curr Opin Clin Nutr Metab Care 2015; 18(3):254-262.

33. Martins MC, Oliveira FCA, Silveira ACB, Gonzaga KBC, Xagoraris M, Centeno JR, Souza JAC. Importância da avaliação bioquímica mensal na triagem de pacientes com desnutrição em hemodiálise. J Bras Nefrol 2010; 32(4):352-358.

34. Stolic R. Obesity in renal failure--health or disease? Med Hypotheses 2010; 75(6):497-500.

35. Brasil. Ministério da Saúde (MS). Vigitel Brasil 2014. Vigilância de Fatores de Risco e proteção para doenças crônicas por inquérito telefônico. Brasília: MS; 2014.

36. Collins AJ, Foley RN, Gilbertson DT, Chen SC. United States renal data system public health surveillance of chronic kidney disease and end-stage renal disease. Kidney Int Suppl 2015; 5(1):2-7.

37. Coelho VSP. Interesses e instituições na política de saúde. Rev Bras Ciências Sociais 1998; 13(37):115-128.

38. Nardino D, Krüger TR. O serviço privado contratualizado pelo SUS: o caso da diálise. Rev Grifos 2007; 16(22/23):209-318.

39. Cobo G, Hecking M, Port FK, Exner I, Lindholm B, Stenvinkel P, Carrero JJ. Sex and gender differences in chronic kidney disease: progression to end-stage renal disease and haemodialysis. Clin Sci (Lond) 2016; 130(14):1147-1163.

40. Neugarten, J., Acharya, A. and Silbiger, S.R. Effect of gender on the progression of nondiabetic renal disease: a meta-analysis. J Am Soc Nephrol 2000; 11(2):319-329.

41. Pscheidt C, Nagel G, Zitt E, Kramar R, Concin H, Lhotta K. Sex- and time-dependent patterns in risk factors of end-stage renal disease: a large Austrian cohort with up to 20 years of follow-up. PloS One 2015; 10:e135052.

42. Crews DC, Kuczmarski MF, Miller ER, Zonderman AB, Evans MK, Powe NR. Dietary habits, poverty, and chronic kidney disease in an urban population. J Ren Nutr 2015; 25(2):103-110.

43. Verhave JC, Hillege HL, Burgerhof JG, Navis G, de Zeeuw D, de Jong PE. Cardiovascular risk factors are differently associated with urinary albumin excretion in men and women. J Am Soc Nephrol 2003; 14(5):1330-1335.

Artigo apresentado em 03/07/2016

Aprovado em 30/06/2017

Versão final apresentada em 02/07/2017 
